\title{
Cost Sharing and Strategyproof Mechanisms for Set Cover Games
}

\author{
Xiang-Yang Li ${ }^{\star 1}$, Zheng Sun ${ }^{\star \star 2}$, and WeiZhao Wang ${ }^{1}$ \\ 1 Illinois Institute of Technology, Chicago, IL, USA, \{lixian, wangwei4\} @iit .edu \\ 2 Hong Kong Baptist University, Hong Kong, China, sunz@ comp. hkbu . edu . hk
}

\begin{abstract}
We develop for set cover games several general cost-sharing methods that are approximately budget-balanced, core, and/or group-strategyproof. We first study the cost sharing for a single set cover game, which does not have a budget-balanced core. We show that there is no cost allocation method that can always recover more than $\frac{1}{\ln n}$ of the total cost if we require the cost sharing being a core. Here $n$ is the number of all players to be served. We give an efficient cost allocation method that always recovers $\frac{1}{\ln d_{\max }}$ of the total cost, where $d_{\max }$ is the maximum size of all sets. We then study the cost allocation scheme for all induced subgames. It is known that no cost sharing scheme can always recover more than $\frac{1}{n}$ of the total cost for every subset of players. We give an efficient cost sharing scheme that always recovers at least $\frac{1}{2 n}$ of the total cost for every subset of players and furthermore, our scheme is cross-monotone. When the elements to be covered are selfish agents with privately known valuations, we present a strategyproof charging mechanism, under the assumption that all sets are simple sets, such that each element maximizes its profit when it reports its valuation truthfully; further, the total cost of the set cover is no more than $\ln d_{\max }$ times that of an optimal solution. When the sets are selfish agents with privately known costs, we present a strategyproof payment mechanism in which each set maximizes its profit when it reports its cost truthfully. We also show how to fairly share the payments to all sets among the elements.
\end{abstract}

\section{Introduction}

Generalized Set Cover Problem Let $U=\left\{e_{1}, e_{2}, \cdots, e_{n}\right\}$ be a finite set, and let $\mathcal{S}=$ $\left\{S_{1}, S_{2}, \cdots, S_{m}\right\}$ be a collection of multisets (or sets for short) of $U$. For each $e_{i} \in U$ and each $S_{j} \in \mathcal{S}$, we denote the multiplicity of $e_{i}$ in $S_{j}$ by $k_{j, i}$. Each $S_{j}$ is associated with a cost $c_{j}$. For any $\mathcal{X} \subseteq \mathcal{S}$, let $C(\mathcal{X})$ denote the total costs of the sets in $\mathcal{X}$, i.e., $C(\mathcal{X})=\sum_{S_{j} \in \mathcal{X}} c_{j}$. For a given $k>0$ and a set of element coverage requirements $\left\{r_{1}, r_{2}, \cdots, r_{n}\right\}$, a $k$-partial-cover $\mathcal{C}$ is defined to be a subset $\left\{S_{j_{1}}, S_{j_{2}}, \cdots, S_{j_{l}}\right\}$ of $\mathcal{S}$ such that $\sum_{i=1}^{n} \min \left\{r_{i}, \sum_{t=1}^{l} k_{j_{t}, i}\right\} \geq k$. The generalized set cover problem is to compute an optimum $k$-partial-cover $\mathcal{C}_{\text {opt }}$ with the minimum $\operatorname{cost} C\left(\mathcal{C}_{\text {opt }}\right)$.

\footnotetext{
* The research of the author was supported in part by NSF under Grant CCR-0311174. The research was conducted when the author was visiting Hong Kong Baptist University.

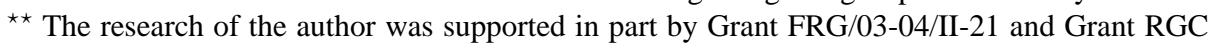
HKBU2107/04E.
} 
This problem becomes the traditional multicover problem [1,2] when we set $k=$ $\sum_{i=1}^{n} r_{i}$ and $k_{j, i}=1$ for all $S_{j}$ and $e_{i}$, as each element $e_{i}$ should be fully covered and each set $S_{j}$ is a simple set. When we set $r_{i}=1$, it becomes the traditional partial cover problem [3]. This problem is therefore a natural extension of the classic set cover problem by allowing partial cover, multiset, and element coverage requirement greater than 1. Accordingly, the greedy algorithm for this problem is a combination of the algorithms designed for partial cover and multicover [1-3].

Set Cover Game Consider the following scenario: a company can choose from a set of service providers $\mathcal{S}=\left\{S_{1}, S_{2}, \cdots, S_{m}\right\}$ to provide services to a set of service receivers $U=\left\{e_{1}, e_{2}, \cdots, e_{n}\right\}$.

- With a fixed cost $c_{j}$, each service provider $S_{j}$ can provide services to a fixed subset of service receivers.

- There may be a limit $k_{j, i}$ on the number of units of service that a service provider $S_{j}$ can provide to a service receiver $e_{i}$. For example, each service provider may be a cargo company that is transporting goods to various cities (the service receivers), and the amount of goods that can be transported to a particular city daily is limited by the number of trains/trucks that are going to that city everyday.

- Each service receiver $e_{i}$ may have a limit $r_{i}$ on the number of units of service that it desires to receive (and is willing to pay for).

- There may be a limit $k$ on the total number of units of service that the service providers shall provide to the service receivers.

The problem can be modeled by the generalized set cover problem defined previously. There may be different types of games according to various conditions:

1. Each service receiver $e_{i}$ has to receive at least $r_{i}$ units of service, and the costs incurred by the service providers will be shared by the service receivers.

2. Each service receiver $e_{i}$ declares a bid $b_{i, r}$ for the $r$-th unit of service it shall receive, and is willing to pay for it only if the assigned cost is at most $b_{i, r}$.

3. Each service provider $S_{j}$ declares a cost $c_{j}$, and is willing to provide the service only if the payment received is at least $c_{j}$.

There are different algorithmic issues for these games. For example, for Game 1, we shall define a cost allocation method so that every subset of service receivers feel that the total cost they need to pay is "fair" according to certain criteria. For Games 1 and 2, the cost allocation method, by charging service receivers, needs to recover (either entirely or a constant fraction of) the total cost of the chosen service providers. For Games 2 and 3, we need a mechanism (for determining costs charged to service receivers and payments paid to service providers) that can guarantee that the players are truthful with their declaration of bids/costs.

Our Results We first study how we share the cost of the selected service providers among the service receivers such that some fairness criteria are met. We present a cost sharing method that is $\frac{1}{\ln d_{\max }}$-budget-balanced and core, where $d_{\max }$ is the maximum set size. The bound $\frac{1}{\ln d_{\max }}$ is tight. We also present a cost sharing method that is $\frac{1}{2 n}$ budget-balanced core and cross-monotone, which is almost the optimum [4].

We then design greedy set cover methods that are cognizant of the fact that the service providers or the service receivers are selfish and rational. By "selfish," we mean that they only care about their own benefits without consideration for the global perfor- 
mances or fairness issues. By "rational," we mean that when the methods of computing the output for the set cover game are instituted, they will always choose their actions to maximize their benefits. When the elements to be covered are selfish agents with privately known valuations, we present a strategyproof charging mechanism, under the assumption that all sets are simple sets, such that each element maximizes its profit when it reports its valuation truthfully; further, the total cost of the set cover is no more than $\ln d_{\max }$ times that of an optimal solution for these selected service receivers and their coverage requirements. When the sets are selfish agents with privately known costs, we present a strategyproof payment mechanism in which each set maximizes its profit when it reports its cost truthfully. We also show how to fairly share the payments to all sets among the elements.

Paper Organization In Section 2, we give the exact definitions of fair cost sharing and mechanism design. In Section 3, we study how to fairly share the cost of the service providers among the covered service receivers when the receivers must receive the service. We show in Section 4 how to charge the cost of service providers to the selfish service receivers when each receiver has a valuation on the $r$-th cover received. We then show in Section 5 a strategyproof payment scheme to the selfish service providers when each has a privately known cost. We conclude our paper in Section 6.

\section{Preliminaries and Prior Art}

\subsection{Preliminaries}

Algorithm Mechanism Design (AMD) Assume that there are $n$ agents. Each agent $i$, for $i \in\{1, \cdots, n\}$, has some private information $t_{i}$, called its type. All agents' types define a type vector $t=\left(t_{1}, t_{2}, \cdots, t_{n}\right)$. A mechanism defines, for each agent $i$, a set of strategies $A_{i}$. For each strategy vector $a=\left(a_{1}, \cdots, a_{n}\right)$, i.e., agent $i$ plays a strategy $a_{i} \in A_{i}$, the mechanism computes an output o $=\mathcal{O}(a)$ and a payment vector $\mathcal{P}(a)=\left(p_{1}, \cdots, p_{n}\right)$, where $p_{i}=\mathcal{P}_{i}(a)$ is the amount of money given to the participating agent $i$. Let $v_{i}\left(t_{i}, o\right)$ denote agent $i$ 's preferences to an output $o$ and $u_{i}\left(t_{i}, o(a), p_{i}(a)\right)$ denote its utility at the outcome $(o, p)$ of the game. We assume that agents are rational and have quasi-linear utility functions. The utility function is quasilinear if $u_{i}\left(t_{i}, o\right)=v_{i}\left(t_{i}, o\right)+p_{i}$. An agent is called rational if it always adopts its best strategy (called dominant strategy) that maximizes its utility regardless of what other agents do. A direct-revelation mechanism is incentive compatible (IC) if reporting valuation truthfully is a dominant strategy. Another common requirement in the literature for mechanism design is the so called individual rationality (IR): the agent's utility of participating in the output of the mechanism is not less than the utility of the agent if it did not participate at all. A mechanism is called truthful or strategyproof if it satisfies both IC and IR properties. To make the mechanism tractable, both methods $\mathcal{O}()$ and $\mathcal{P}()$ should be computable in polynomial time. A mechanism $M=(\mathcal{O}, \mathcal{P})$ is $\beta$ efficient if $\forall t, \sum_{i=1}^{n} v_{i}\left(t_{i}, \mathcal{O}(t)\right) \geq \beta \cdot \max _{o} \sum_{i=1}^{n} v_{i}\left(t_{i}, o\right)$. Obviously for the set cover game, we cannot design an $o(\ln n)$-efficient polynomial-time computable strategyproof mechanism unless $N P \subset D T I M E\left(n^{\log \log n}\right)$ [2]. 
Cost Sharing Consider a set $U$ of $n$ players. For a subset $S \subseteq U$ of players, let $C(S)$ be the cost of providing service to $S$. Here $C(S)$ could be the minimum cost, denoted by $\operatorname{OPT}(S)$, or the cost computed by some algorithm $\mathcal{A}$, denoted by $\mathcal{A}(S)$. We always assume that the cost function $C(S)$ is cohesive, i.e., for any two disjoint subsets $S_{1}$ and $S_{2}, C\left(S_{1} \cup S_{2}\right) \leq C\left(S_{1}\right)+C\left(S_{2}\right)$. A cost sharing scheme is simply a function $\xi(i, S)$ with $\xi(i, S)=0$ for $i \notin S$, for every set $S \subseteq U$ of players. An obvious criterion is that the sharing method should be fair. While the definition of budget-balance is straightforward, defining fairness is more subtle: many fairness concepts were proposed in the literature, such as max-min [5], min-max [6], core and bargaining set [7]. Typically, the following three properties are required by a cost sharing scheme.

1. ( $\alpha$-budget-balance) For some given parameter $\alpha \leq 1, \alpha \cdot C(U) \leq \sum_{i \in U} \xi(i, U) \leq$ $C(U)$. If $\alpha=1$, we call the cost sharing scheme budget-balanced.

2. (fairness under core) For any subset $S \subseteq U, \sum_{i \in S} \xi(i, U) \leq \mathrm{OPT}(S)$.

3. (Cross-monotonicity) For any two subsets $S \subseteq T$ and $i \in S, \xi(i, S) \geq \xi(i, T)$.

When only the first two conditions are satisfied, we call the cost sharing scheme to be in the $\alpha$-core. When all three conditions are met, we call the cost sharing scheme to be cross-monotone $\alpha$-core. When each player $i$ has a valuation $v_{i}$ on getting the service, a mechanism should first decide the output of the game (who will get the service), and then decide what is the share of each selected player (what is the payment method). It is well-known that a cross-monotone cost sharing scheme implies a group-strategyproof mechanism [8]. Notice that the cross-monotone property is not the necessary condition for group-strategyproof. Naturally, several additional properties are required for a cost sharing scheme when every player has a valuation on getting the service.

1. (Incentive Compatibility) Assume that the valuation by player $i$ on getting the service is $v_{i}$. Let $b=\left(b_{1}, b_{2}, \cdots, b_{n}\right)$ be the bidding vector of $n$ players. Let $\mathcal{O}(b)=\left(o_{1}, o_{2}, \cdots, o_{n}\right)$ denote whether a player is selected to get the service or not and $\mathcal{P}(b)$ be the charge to player $i$, i.e., the mechanism is $M=(\mathcal{O}(b), \mathcal{P}(b))$. It satisfies IC if every player maximizes its profit $v_{i} \cdot o_{i}-p_{i}$ when $b_{i}=v_{i}$.

2. (No Positive Transfer) For every player $i, p_{i} \geq 0$.

3. (Individual Rationality) For every player $i, v_{i} \cdot o_{i}-p_{i} \geq 0$.

4. (Consumer Sovereignty) Fix the bids of all other players, there exists a value $\tau_{i}$ such that player $i$ is guaranteed to get the service when its bid is larger than $\tau_{i}$.

\subsection{Prior Arts on Cost Sharing and Algorithm Mechanism Design}

Although the traditional set cover problem (without multisets and partial-cover requirement) can be viewed as a special case of multicast, several results were proposed specifically for set cover in selfish environment. Devanur et al. [9] studied, for the set cover game and facility location game, how the cost of shared resource is to be distributed among its users in such a way that revealing the true valuation is a dominant strategy for each user. Their cost sharing method is not in the core of the game. One of the open questions left in [9] is to design a strategyproof cost sharing method for multicover game in which the bidders might want to get covered multiple times. For facility location game, Pál and Tardos [10] gave a cost sharing method that can recover $\frac{1}{3}$ of the total cost, and recently, Immorlica et al. [4] showed that this is the best achievable upper 
bound for any cross-monotonic cost sharing method. Sharing the cost of the multicast structure among receivers was studied in $[8,11-16]$ so some fairness is accomplished.

\section{Cost Sharing Among Unselfish Service Receivers}

In this section, we study how to share the cost of the service providers among a given set of service receivers. For this scenario, it is difficult to find realistic examples where a partial cover is desired. Therefore, in the remainder of this section, we only consider the multiset multicover problem, i.e., $k=\sum_{i=1}^{n} r_{i}$. However, the results presented here can easily be generalized to the partial cover case, should such a scenario arise.

\section{$3.1 \alpha$-Core}

Given a subset of elements $X$, let $\operatorname{OPT}(X)$ denote the cost of an optimum cover $\mathcal{C}_{\text {opt }}(X)$ of $X$. This cost function clearly is cohesive: for every partition $T_{1}, T_{2}, \cdots$, $T_{t}$ of $U, \mathrm{OPT}(U) \leq \sum_{i=1}^{t} \mathrm{OPT}\left(T_{i}\right)$. A cost allocation for $U$ is a $n$-dimensional vector $x=\left(x_{1}, x_{2}, \cdots, x_{n}\right)$ that specifies for each element $e_{i} \in U$ the share $x_{i} \geq 0$ of the total cost of serving $U$ that $e_{i}$ shall pay. Ideally, when the set of elements to be covered is fixed to be $U$, we want the cost allocation $x$ to be budget-balanced and fair, i.e., being in core. However, a simple example in [17] shows that there is no budget-balanced core for the set-cover game. We then relax the notion of budget-balance to the notion of $\alpha$-budget-balance for some $\alpha \leq 1$. See [17] for the proof of the achievable $\alpha$-core.

Theorem 1. For the set cover game, no cost allocation method is $\alpha$-core for $\alpha>\frac{1}{\ln n}$.

We then give a cost allocation method that can recover $\frac{1}{\ln d_{\max }}$ of the total cost $\mathrm{OPT}(U)$ for a multiset multicover game, where $d_{\max }=\max _{1 \leq j \leq m}\left|S_{j}\right|$. Without loss of generality, we assume that $d_{\max } \leq \sum_{i=1}^{n} r_{i}$. The basic approach of our cost allocation method is as follows. We first run the greedy Algorithm 1 to find a set cover $\mathcal{C}_{\text {grd }}$ with an approximation ratio of $\ln d_{\max }$. Starting with $\mathcal{C}_{\text {grd }}=\emptyset$, the greedy algorithm adds to $\mathcal{C}_{g r d}$ a set $S_{j_{t^{\prime}}}$ at each round $t^{\prime}$. After the $s$-th round, we define the remaining required coverage $r_{i}^{\prime}$ of an element $e_{i}$ to be $r_{i}-\sum_{t^{\prime}=1}^{s} k_{j_{t^{\prime}}, i}$. For any $S_{j} \notin \mathcal{C}_{g r d}$, the effective coverage $k_{j, i}^{\prime}$ of $e_{i}$ by $S_{j}$ is defined to be $\min \left\{k_{j, i}, r_{i}^{\prime}\right\}$, the value $v_{j}$ of $S_{j}$ is defined to be $\sum_{i=1}^{n} k_{j, i}^{\prime}$, and the effective average cost of $S_{j}$ is defined to be $\frac{c_{j}}{v_{j}}$.

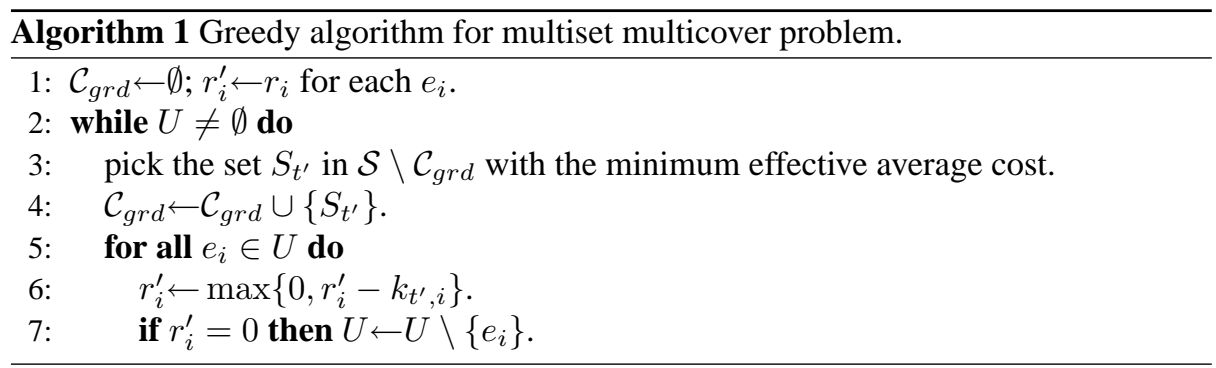


The greedy algorithm will select a set $S_{j}$ with the least effective average cost. For any $e_{i}$ and $r$ such that $r_{i}-r_{i}^{\prime}+1 \leq r \leq r_{i}-r_{i}^{\prime}+k_{j, i}^{\prime}$, we let price $(i, r)=\frac{c_{j}}{v_{j}}$. Let $x_{i}^{\prime}=\sum_{r=1}^{r_{i}} \operatorname{price}(i, r)$ and $x_{i}=\frac{x_{i}^{\prime}}{\ln d_{\max }}$. We claim the following theorem (see [17]):

Theorem 2. The above-defined cost allocation $x$ is a $\frac{1}{\ln d_{\max }}$-core.

Recall that the core we defined, given a set of players $U$, required that $\sum_{e_{i} \in T} \xi(i, U)$ is at most the optimum cost of providing service to elements in $T$. For a set cover game, clearly it is NP-hard to find the optimum cost of covering $T$. Naturally, one may relax the $\alpha$-core as follows: a cost sharing method $\xi(i, \cdot)$ is called a relaxed $\alpha$-core if (1) $\alpha \cdot \mathcal{C}_{\text {grd }}(U) \leq \sum_{i \in U} \xi(i, U) \leq \mathcal{C}_{\text {grd }}(U)$; and (2) $\sum_{i \in T} \xi(i, U) \leq \mathcal{C}_{\text {grd }}(T)$ for every subset $T \subseteq U$. Even we relax the definition of the core to this, we can still prove in [17] that with the cost function computed by the greedy algorithm, there is no cost sharing method that is a relaxed $\alpha$-core for $\alpha=\Omega\left(\frac{1}{\ln n}\right)$.

\subsection{Cross-monotone $\alpha$-Core}

Clearly, if a cost sharing scheme is cross-monotone $\alpha$-core then every cost allocation method $\xi(\cdot, S)$ induced on a subset $S$ of players is always $\alpha$-core, but the reverse is not true. From Theorem 1, clearly no cost sharing scheme for the set cover game is crossmonotone $\alpha$-core for $\alpha=\frac{1}{\ln n}$. Recently, it was claimed in [4] that for set cover game, there is no cross-monotone $\alpha$-core cost sharing scheme for $\alpha=\frac{1}{n}+\epsilon$.

For generalized set cover games, we will present a cross-monotone cost sharing scheme $\xi(i, S)$ (see Algorithm 2) that can recover $\frac{1}{2 n}$ of the total cost. We show an example in [17] that the bound $\frac{1}{2 n}$ is tight for Algorithm 2. Further, the bound is tight, for set cover games without multisets (but still allowing multicover requirements): our cross-monotone cost sharing scheme $\xi(i, S)$ can recover $\frac{1}{n}$ of the total cost.

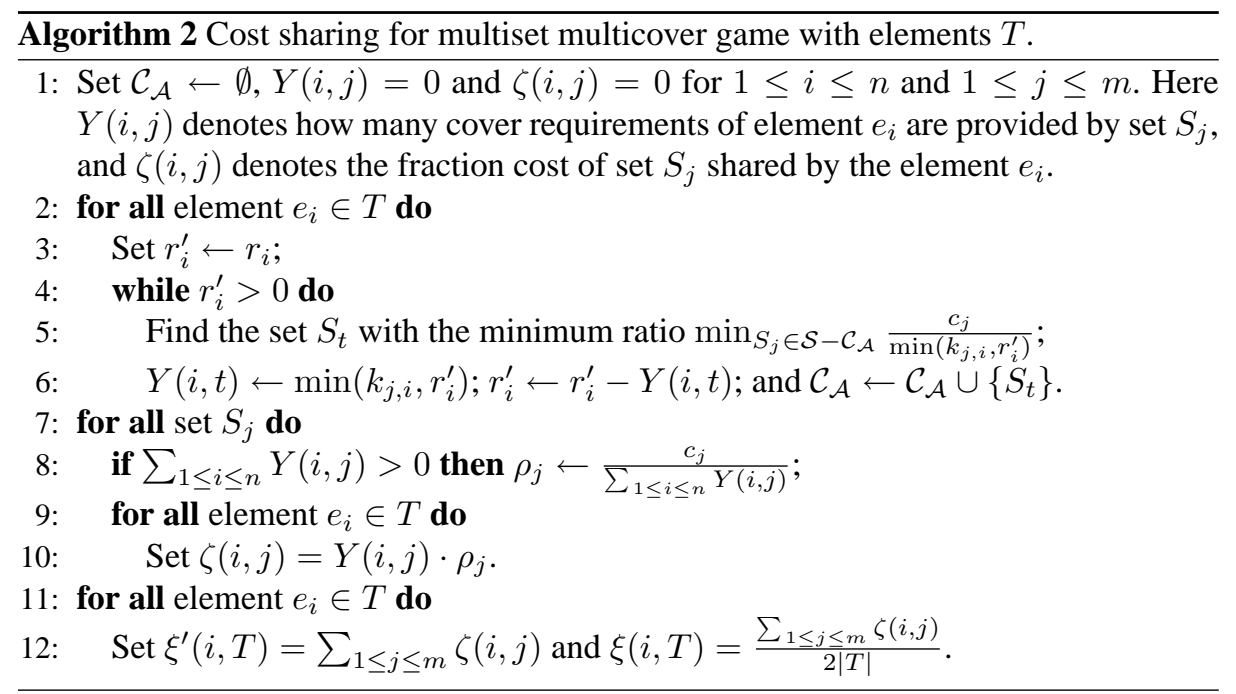


Theorem 3. The cost sharing scheme $\xi(\cdot, \cdot)$ is a cross-monotone $\frac{1}{2 n}$-core and is crossmonotone $\frac{1}{n}$-core for set cover game when every set $S_{j}$ is a simple set.

\section{Cost Sharing Among Selfish Service Receivers}

In Section 3 we assumed that all elements (service receivers) are unselfish and all their coverage requirements are to be satisfied. In this section, we consider the problem of selecting service providers under the constraint of a collection of bids $B=B_{1} \cup B_{2} \cup$ $\cdots \cup B_{n}$. Each $B_{i}$ contains a series of bids $b_{i, 1}, b_{i, 2}, \cdots, b_{i, r_{i}}$, where $b_{i, r}$ denotes the declared price that element $e_{i}$ is willing to pay for the $r$-th coverage (i.e., the valuation of the $r$-th coverage). In this scenario, we may also consider partial cover, as the total number of units of service available may be limited by a constant $k$.

We assume that $b_{i, 1} \geq b_{i, 2} \geq \cdots \geq b_{i, r_{i}}$. This is often true in realistic situations: the marginal valuations are usually decreasing. A bid $b_{i, r}$ will be served (and the subsequent bid $b_{i, r+1}$ will be considered) only if $b_{i, r} \geq \operatorname{price}(i, r)$, where price $(i, r)$ is the cost to be paid by $e_{i}$ for its $r$-th coverage. Further, to guarantee that the mechanism is both strategyproof and budget-balanced, we assume that each set is a simple set.

We use a greedy algorithm (see Algorithm 3) similar to the one for the traditional set cover game [9]. Informally speaking, we start with $y=0$, where $y$ is the cost to be shared by each bid served. We raise $y$ until there exists a set $S_{j}$ whose cost can be sufficiently covered by the element copies in $S_{j}$, if each element copy needs to pay $y$. To adapt to the multicover scenario, we make the following changes:

$\star$ For any set $S_{j} \notin \mathcal{C}_{\text {grd }}$ and any $e_{i}$, we define the collection of alive bids $B_{i}^{(j)}$ of $e_{i}$ with respect to $S_{j}$ to be $\left\{b_{i, r_{i}-r_{i}^{\prime}+1}\right\}$ if $k_{j, i}^{\prime}>0$ (i.e., $k_{j, i}^{\prime}=1$ since $S_{j}$ is a simple set) and $b_{i, r_{i}-r_{i}^{\prime}+1} \geq y$, or $\emptyset$ if otherwise. That is, if $y$ is the cost to be paid for each bid served, $B_{i}^{(j)}$ contains the bid of $e_{i}$ covered by $S_{j}$ that can afford the cost (if any). $\star$ Define the value $v_{j}$ of $S_{j}$ as $\sum_{i=1}^{n}\left|B_{i}^{(j)}\right|$, and its effective average cost as $\frac{c_{j}}{v_{j}}$.

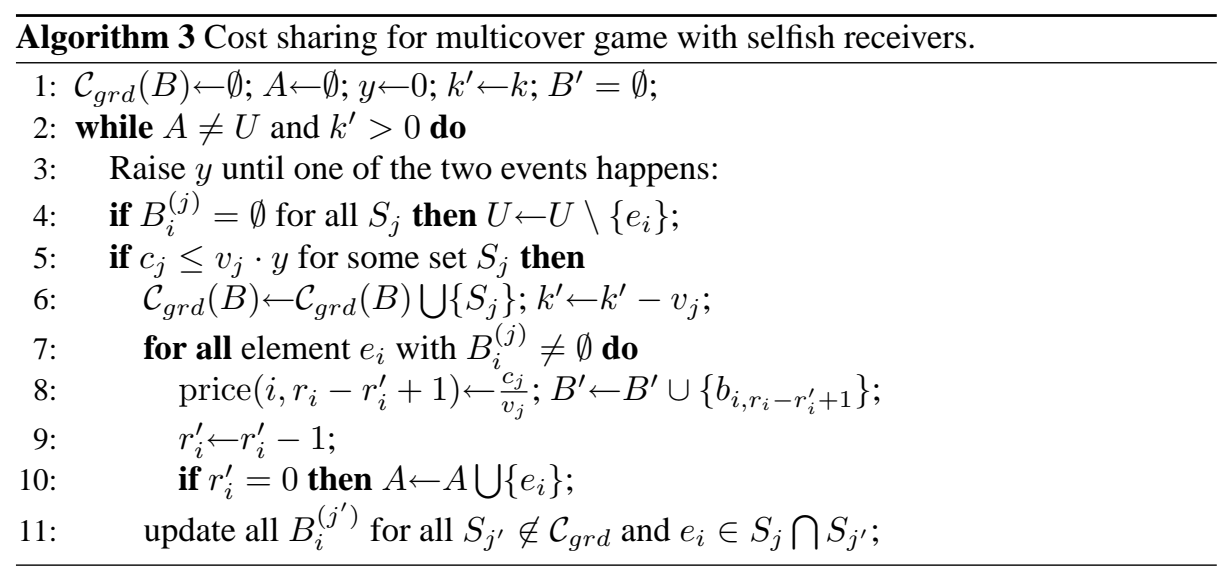


When the algorithm terminates, $B^{\prime}$ contains all bids (of all elements) that are served. We prove the following theorem about this mechanism (see [17] for proof):

Theorem 4. Algorithm 3 defines a strategyproof mechanism. Further, the total cost of the sets selected is no more than $\ln d_{\max }$ times that of an optimal solution.

In [9] multicover game was also considered. However, the algorithm used is different from ours and also they did not assume that the bids by the same element are nonincreasing, and their mechanism is not strategyproof.

\section{Selfish Service Providers}

The underline assumption made so far in previous sections is that the service providers are truthful in revealing their costs of providing the service. In this section, we will address the scenario when service providers are selfish in revealing their costs.

\subsection{Strategyproof Mechanism}

We want to find a subset of agents $D$ such that $\bigcup_{j \in D} S_{j}$ has $r_{i}$ copies of element $e_{i}$ for every element $e_{i} \in U$. Let $c=\left(c_{1}, c_{2}, \cdots, c_{m}\right)$. The social efficiency of the output $D$ is $-\sum_{j \in D} c_{j}$, which is the objective function to be maximized. Clearly a VCG mechanism [18-20] can be applied if we can find the subset of $\mathcal{S}$ that satisfies the multicover requirement of elements in $U$ with the minimum cost. Unfortunately this is NP-hard. Let $\mathcal{C}_{\text {grd }}(\mathcal{S}, c, U, r)$ be the sets selected from $\mathcal{S}$ (with cost specified by a cost vector $\left.c=\left(c_{1}, \cdots, c_{m}\right)\right)$ by the greedy algorithm to cover elements in $U$ with cover requirement specified by a vector $r=\left(r_{1}, \cdots, r_{n}\right)$ (see Algorithm 1). We assume that the type of an agent is $\left(S_{j}, c_{j}\right)$, i.e., every service provider $j$ could lie not only about its cost $c_{j}$ but also about the elements it could cover. This problem now looks very similar to the combinatorial auction with single minded bidder studied in [21]. We show in [17] that the mechanism $M=\left(\mathcal{C}_{g r d}, \mathcal{P}^{V C G}\right)$ is not truthful, i.e., use Algorithm 1 to find a set cover, and apply VCG mechanism to compute the payment to the selected agents: the payment to an agent $j$ is 0 if $S_{j} \notin \mathcal{C}_{g r d}$; otherwise, the payment to a set $S_{j} \in \mathcal{C}_{\text {grd }}$ is $\mathcal{P}_{j}^{V C G}=C\left(\mathcal{C}_{\text {grd }}\left(\mathcal{S} \backslash\left\{S_{j}\right\},\left.c\right|^{j} \infty, U, r\right)\right)-C\left(\mathcal{C}_{\text {grd }}(\mathcal{S}, c, U, r)\right)+c_{j}$. Here $C(\mathcal{X})$ is the total cost of the sets in $\mathcal{X} \subseteq \mathcal{S}$.

For the moment, we assume that agent $j$ won't be able to lie about its element $S_{j}$. We will drop this assumption later. Clearly, the greedy set cover method presented in Algorithm 1 satisfies a monotone property: if a set $S_{j}$ is selected with a cost $c_{j}$, then it is still selected with a cost less than $c_{j}$. Monotonicity guarantees that there exists a strategyproof mechanism for generalized set cover games using Algorithm 1 to compute its output. We then show how to compute the payment to each service provider efficiently. We assume that for any set $S_{j}$, if we remove $S_{j}$ from $\mathcal{S}, \mathcal{S}$ still satisfies the coverage requirements of all elements in $U$. Otherwise, we call the set cover problem to be monopoly: the set $S_{j}$ can charge an arbitrarily large cost in the monopoly game. The following presents our payment scheme for multiset multicover set cover problem.

We show in [17] that the mechanism $M=\left(\mathcal{C}_{\text {grd }}, \mathcal{P}^{\text {grd }}\right)$ is strategyproof (when the agent $j$ does not lie about the set $S_{j}$ of elements it can cover) and the payment $\mathcal{P}_{j}^{\text {grd }}$ 


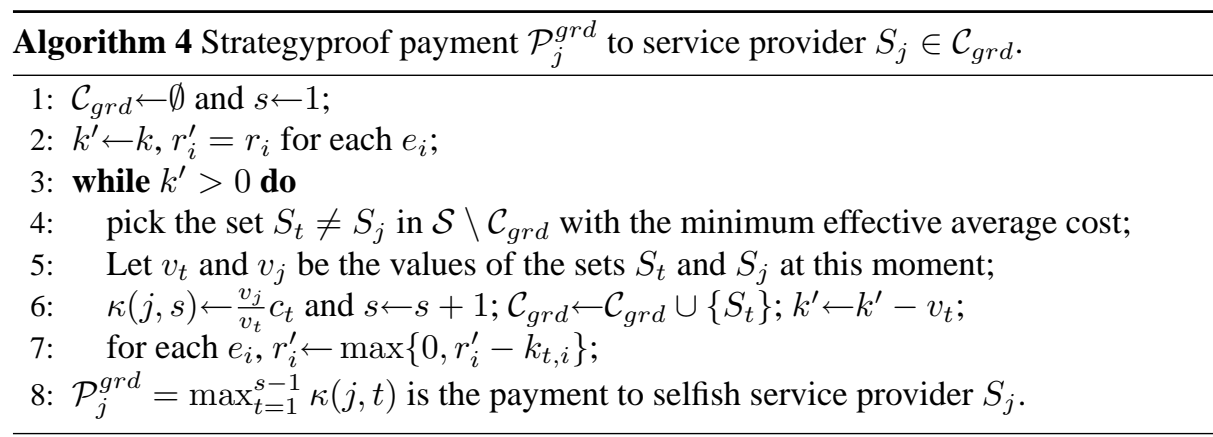

is the minimum to the selfish service provider $j$ among any strategyproof mechanism using Algorithm 1 as its output. We now consider the scenario when agent $j$ can also lie about $S_{j}$. Assume that agent $j$ cannot lie upward ${ }^{3}$, i.e., it can only report a $S_{j}^{\prime} \subseteq S_{j}$. We argue that agent $j$ will not lie about its elements $S_{j}$. Notice that the value $\kappa(j, s)$ computed for the $s$-th round is $\kappa(j, s)=\frac{v_{j}}{v_{t}} c_{t}=\frac{\sum_{1 \leq i \leq n} \min \left(r_{i}^{\prime}, k_{j, i}\right)}{\sum_{1 \leq i \leq n} \min \left(r_{i}^{\prime}, k_{t, i}\right)} c_{t}$. Obviously $v_{j}$ cannot increase when agent $j$ reports any set $S_{j}^{\prime} \subseteq S_{j}$. Thus, falsely reporting a smaller set $S_{j}^{\prime}$ will not improve the payment of agent $j$.

Theorem 5. Algorithm 1 and 4 together define a $\ln d_{\text {max }}$-efficient strategyproof mechanism $M=\left(\mathcal{C}_{\text {grd }}, \mathcal{P}^{\text {grd }}\right)$ for multiset multicover set cover game.

\subsection{Sharing the Payment Fairly}

In the previous subsection, we only define what is the payment to a selfish service provider $S_{j}$. A remaining question is how the payment should be charged fairly (under some subtle definitions) to encourage cooperation among service receivers. One natural way of defining fair payment sharing is to extend the fair cost sharing method. Consider a strategyproof mechanism $M=(\mathcal{O}, \mathcal{P})$. Let $\mathcal{P}(T)$ be the total payment to the selfish service providers when $T$ is the set of service receivers to be covered. A payment sharing scheme is simply a function $\pi(i, T)$ such that $\pi(i, T)=0$ for any element $e_{i} \notin T$. A payment sharing scheme is called $\alpha$-budget-balanced if $\alpha \cdot \mathcal{P}(T) \leq \sum_{e_{i} \in T} \pi(i, T) \leq$ $\mathcal{P}(T)$. A payment sharing scheme is said to be a core if $\sum_{e_{i} \in S} \pi(i, T) \leq \mathcal{P}(S)$ for any subset $S \subset T$. A payment sharing scheme is said to be an $\alpha$-core if it is $\alpha$-budgetbalanced and it is a core. For payment method $\mathcal{P}^{g r d}$, we prove in [17] that

Theorem 6. There is no $\alpha$-core payment sharing scheme for $\mathcal{P}^{\text {grd }}$ if $\alpha>\frac{1}{\ln n}$.

It is easy to show that if we share the payment to a service provider equally among all service receivers covered by this set, the scheme is not in the core of the game. We leave it as an open problem whether we can design an $\alpha$-core payment sharing scheme for the payment $\mathcal{P}^{g r d}$ with $\alpha=O\left(\frac{1}{\ln n}\right)$.

\footnotetext{
${ }^{3}$ This can be achieved by imposing a large enough penalty if an agent could not provide the claimed service when it is selected.
} 
In the next, we study the cross-monotone payment sharing scheme. A payment sharing scheme is said to be cross-monotone if $\pi(i, T) \leq \pi(i, S)$ for any two subsets $S \subset T$ and $i \in S$. A payment sharing scheme is said to be a cross-monotone $\alpha$-core if it is $\alpha$-budget-balanced and cross-monotone, and it is a core. We propose the following conjecture.

Conjecture 1 For the strategyproof mechanism $M=\left(\mathcal{C}_{\text {grd }}, \mathcal{P}^{\text {grd }}\right)$ of a set cover game, there is no payment sharing scheme $\pi(\cdot, \cdot)$ that is cross-monotone $\alpha$-core for $\alpha=\frac{1}{n}+\epsilon$.

In the remaining of this section we will present a cross-monotone budget-balanced payment sharing scheme for a strategyproof payment scheme of the set cover game. Our payment sharing scheme is coupled with the following least cost set mechanism $M=\left(\mathcal{C}_{l c s}, \mathcal{P}^{l c s}\right)$. It uses the output called least cost set $\mathcal{C}_{l c s}$ (described in Algorithm 5): for each service receiver $e_{i}$, we find the service provider $S_{j}$ with the least cost efficiency $\frac{c_{j}}{\min \left(r_{i}, k_{j, i}\right)}$ to cover the element $e_{i}$. New cost efficient sets are found till the cover requirement of $e_{i}$ is satisfied. The payment (described in Algorithm 6) to a set $S_{j}$ is defined as $\mathcal{P}_{j}^{l c s}=\max _{e_{i} \in U} p_{j}^{i}$, where $p_{j}^{i}$ is the largest cost that $S_{j}$ can declare while $S_{j}$ is still selected to cover $e_{i}$. If the set $S_{j}$ is not selected to cover $e_{i}$, then $p_{j}^{i}=0$.
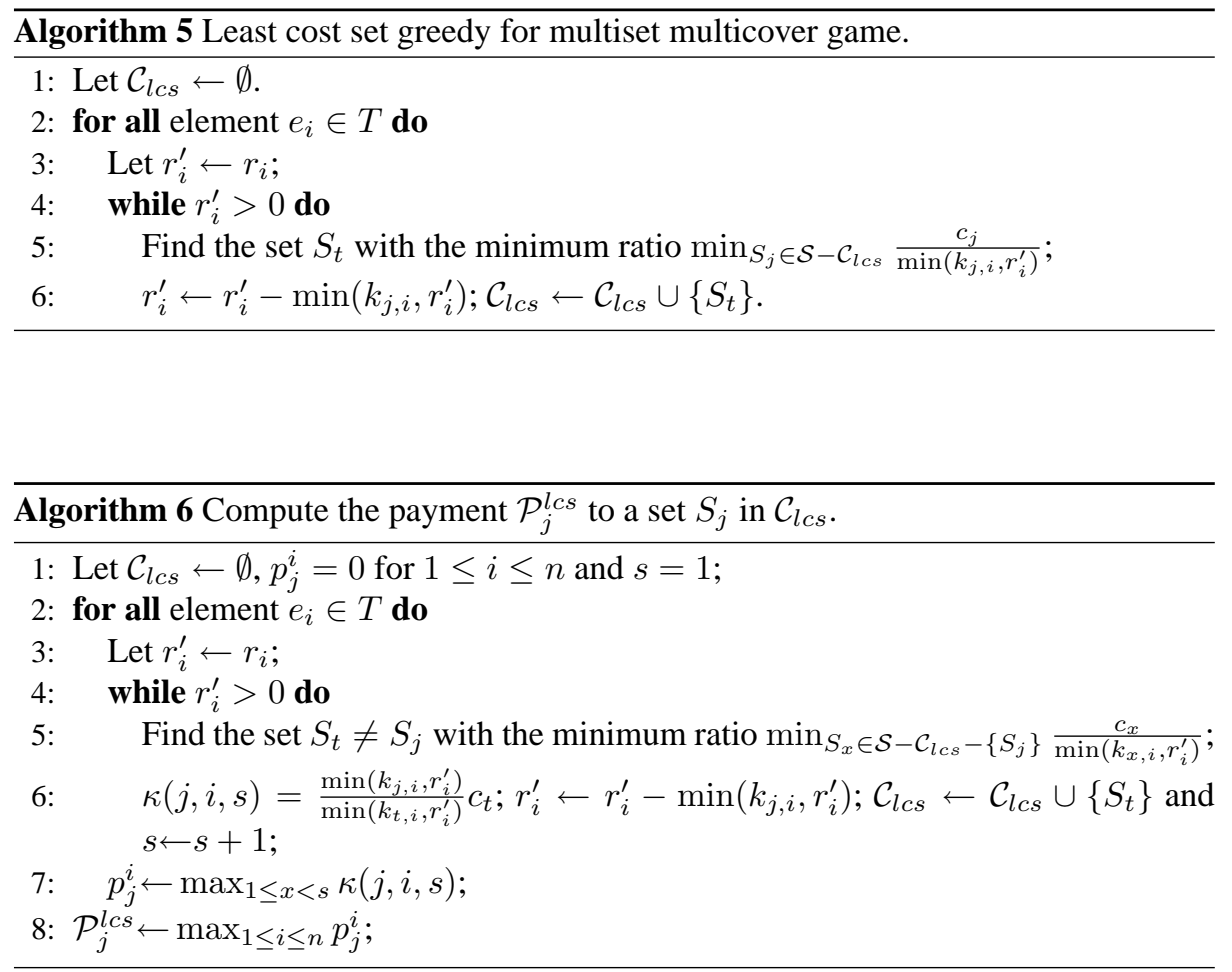

Theorem 7. The mechanism $M=\left(\mathcal{C}_{l c s}, \mathcal{P}^{\text {lcs }}\right)$ is $\frac{1}{2 n}$-efficient and strategyproof. 
We then study how we charge the service receivers so that a budget-balance is achieved and the charging scheme also is fair under some concepts. Notice that, given a subset of elements $T$, we can view the total payments $\mathcal{P}(T)$ to all service providers covering $T$ as a "cost" to $T$. The payment computed by mechanism $M=\left(\mathcal{C}_{l c s}, \mathcal{P}^{l c s}\right)$ clearly is cohesive. Then naturally, we could use the cost-sharing schemes studied before to share this special cost among elements. However, it is easy to show by example that the previous cost-sharing schemes (studied in Section 3) are not in the core and also not cross-monotone.

Roughly speaking, our payment sharing scheme works as follows. Notice that a final payment to a set $S_{j}$ is the maximum of payments $p_{j}^{i}$ by all elements. Since different elements may have different value of payment to set $S_{j}$, the final payment $\mathcal{P}_{j}^{l c s}$ should be shared proportionally to their values, not equally among them as cost-sharing.

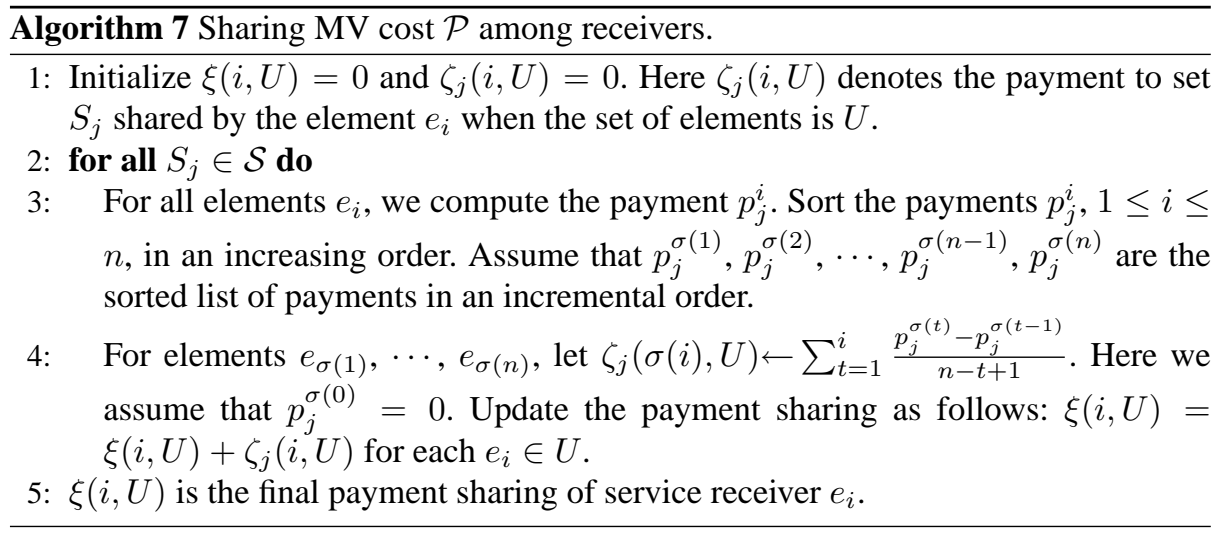

Our payment sharing method described in Algorithm 7 applies to a more general cost function. A cost function $\mathcal{P}$ is said to be maximum-view cost (MV cost) if it is defined as $\mathcal{P}_{j}=\max _{e_{i} \in U} p_{j}^{i}$ where $p_{j}^{i}$ is the view of the cost of set $S_{j}$ by element $e_{i}$. Obviously, the traditional cost $c$ is a MV cost function by setting $p_{j}^{i}=c_{j}$ for each element $e_{i}$. The payment function $\mathcal{P}^{l c s}$ is also a MV cost function.

A service receiver is called free-rider in a payment sharing scheme if its shared total payment is no more than $\frac{1}{n}$ of its total payment it has to pay if it acts alone. Notice that, when a service receiver acts alone, the same mechanism is applied to compute the payment to the service providers.

Theorem 8. The payment sharing scheme described in Algorithm 7 is budget-balanced, cross-monotone, in the core and does not permit free-rider.

\section{Conclusion}

We studied cost sharing and strategyproof mechanisms for various set cover games. We gave an efficient cost allocation method that always recovers $\frac{1}{\ln d_{\max }}$ of the total cost, 
where $d_{\max }$ is the maximum size of all sets. We further gave an efficient cost sharing scheme that is $\frac{1}{2 n}$-budget-balanced, core and cross-monotone. When the elements to be covered are selfish agents with privately known valuations, we presented a strategyproof charging mechanism. When the sets are selfish agents with privately known costs, we presented two strategyproof payment mechanisms in which each set maximizes its profit when it reports its cost truthfully. We also showed how to fairly share the payments to all sets among the elements.

There are a number of open questions left for future research. Are the bounds on the $\alpha$-budget-balanced cost sharing schemes tight, although we proved that they are asymptotically tight? Consider the strategyproof mechanism $M=\left(\mathcal{C}_{\text {grd }}, \mathcal{P}^{\text {grd }}\right)$. Is there a payment sharing method that is $\frac{1}{\ln n}$-core? Is there a payment sharing method that is cross-monotone $\frac{1}{n}$-core? Is this $\frac{1}{n}$ a tight lower bound?

\section{References}

1. Chvátal, V.: A greedy heuristic for the set-covering problem. Mathematics of Operation Research 4 (1979) 233-235

2. Feige, U.: A threshold of for approximating set cover. Journal of the ACM 45 (1998) 634-

3. Slavík, P.: Improved performance of the greedy algorithm for partial cover. Information Processing Letters 64 (1997) 251-254

4. Immorlica, N., Mahdian, M., Mirrokni, V.S.: Limitations of cross-monotonic cost-sharing schemes. In: Proceedings of the 16th Annual ACM-SIAM Symposium on Discrete Algorithms. (2005) To appear.

5. Marbach, P.: Priority service and max-min fairness. IEEE/ACM Transactions on Networking 11 (2003) 733-746

6. Radunović, B., Boudec, J.Y.L. A unified framework for max-min and min-max fairness with applications. In: Proceedings of 40th Annual Allerton Conference on Communication, Control, and Computing. (2002)

7. Osborne, M.J., Rubinstein, A.: A course in game theory. The MIT Press (1994)

8. Moulin, H., Shenker, S.: Strategyproof sharing of submodular costs:budget balance versus efficiency. Economic Theory 18 (2001) 511-533

9. Devanur, N., Mihail, M., Vazirani, V.: Strategyproof cost-sharing mechanisms for set cover and facility location games. In: Proceedings of the 4th ACM Conference on Electronic Commerce. (2003) 108-114

10. Pál, M., Tardos, E.: Group strategyproof mechanisms via primal-dual algorithms. In: Proceedings of the 44th Annual IEEE Symposium on Foundations of Computer Science. (2003) $584-593$

11. Feigenbaum, J., Papadimitriou, C.H., Shenker, S.: Sharing the cost of multicast transmissions. Journal of Computer and System Sciences 63 (2001) 21-41

12. Libman, L., Orda, A.: Atomic resource sharing in noncooperative networks. Telecommunication Systems 17 (2001) 385-409

13. Shenker, S., Clark, D., Estrin, D., Herzog, S.: Pricing in computer networks: Reshaping the research agenda. ACM Computer Communication Review 26 (1996) 19-43

14. Feigenbaum, J., Krishnamurthy, A., Sami, R., Shenker, S.: Hardness results for multicast cost sharing. Theoretical Computer Science 304 (2003) 215-236

15. Archer, A., Feigenbaum, J., Krishnamurthy, A., Sami, R., Shenker, S.: Approximation and collusion in multicast cost sharing. Games and Economic Behavior 47 (2004) 36-71

16. Herzog, S., Shenker, S., Estrin, D.: Sharing the cost of multicast trees: An axiomatic analysis. IEEE/ACM Transactions on Networking 5 (1997) 847-860

17. Li, X.Y., Sun, Z., Wang, W.: Cost sharing and strategyproof mechanisms for set cover games (2004) at http://www.cs.iit.edu/ xli/publications-select.htm.

18. Vickrey, W.: Counterspeculation, auctions and competitive sealed tenders. Journal of Finance 16 (1961) 8-37

19. Clarke, E.H.: Multipart pricing of public goods. Public Choice 11 (1971) 17-33

20. Groves, T.: Incentives in teams. Econometrica 41 (1973) 617-631

21. Lehmann, D.J., Oćallaghan, L.I., Shoham, Y.: Truth revelation in approximately efficient combinatorial auctions. Journal of the ACM 49 (2002) 577-602 